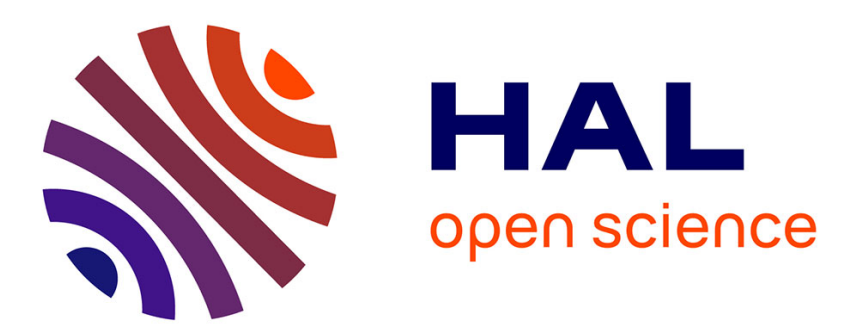

\title{
Rayleigh Waves in Phononic Crystal Made of Multilayered Pillars: Confined Modes, Fano Resonances, and Acoustically Induced Transparency
}

M. Oudich, Bahram Djafari-Rouhani, B. Bonello, Yan Pennec, S. Hemaidia, F. Sarry, D. Beyssen

\section{To cite this version:}

M. Oudich, Bahram Djafari-Rouhani, B. Bonello, Yan Pennec, S. Hemaidia, et al.. Rayleigh Waves in Phononic Crystal Made of Multilayered Pillars: Confined Modes, Fano Resonances, and Acoustically Induced Transparency. Physical Review Applied, 2018, 9 (3), pp.034013. 10.1103/PhysRevApplied.9.034013 . hal-01826538

\section{HAL Id: hal-01826538 \\ https://hal.sorbonne-universite.fr/hal-01826538}

Submitted on 29 Jun 2018

HAL is a multi-disciplinary open access archive for the deposit and dissemination of scientific research documents, whether they are published or not. The documents may come from teaching and research institutions in France or abroad, or from public or private research centers.
L'archive ouverte pluridisciplinaire HAL, est destinée au dépôt et à la diffusion de documents scientifiques de niveau recherche, publiés ou non, émanant des établissements d'enseignement et de recherche français ou étrangers, des laboratoires publics ou privés. 


\title{
Rayleigh Waves In Phononic Crystal Made Of Multilayered Pillars: Confined Modes, Fano Resonances And Acoustic Induced Transparency
}

\author{
M. Oudich ${ }^{1,2}$, B. Djafari-Rouhani ${ }^{3}$, B. Bonello ${ }^{4}$, Y. Pennec ${ }^{3}$, S. Hemaidia ${ }^{1,2}$, F. Sarry ${ }^{1,2}$, D. Beyssen ${ }^{1,2}$ \\ ${ }^{1}$ CNRS, Institut Jean Lamour, UMR 7198, Vandœuvre-lès-Nancy F-54506, France \\ ${ }^{2}$ Université de Lorraine, Institut Jean Lamour, UMR 7198, Vandœuvre-lès-Nancy 54506, France \\ ${ }^{3}$ Institut d'Electronique, de Micro-électronique et de Nanotechnologie (IEMN-UMR CNRS 8520), \\ Université de Lille Sciences et Technologies, Cité Scientifique, 59652 Villeneuve d'Ascq Cedex, France \\ ${ }^{4}$ Sorbonne Universités, UPMC Université Paris 06, INSP UMR CNRS 7588, 4 place Jussieu 75005 Paris,
} France

\begin{abstract}
We present a design of phononic crystal based on pillars distributed on a substrate surface in which each pillar is constructed by a periodic stacking of PMMA and silicon layers. The pillar behaves like a one-dimensional phononic crystal which allows the creation of band gaps that prohibits wave propagation along the pillar. Thanks to this property, we show that confined modes are produced at the pillar-substrate interface which couples with surface acoustic waves (SAW) and causes their attenuation. Furthermore, by tailoring a defect inside the phononic pillar, we reveal the possibility to create confined cavity modes inside the band gap which can strongly couple with SAW. The cavity modes can be excited by SAW and the coupling produces sharp SAW transmissions. Additionally, we demonstrate that the coupling between the cavity modes and the confined modes at the pillar-substrate interface can give rise to a Fano-like resonance. We also evidence the possibility of generating an acoustic analogue of electromagnetically induced transparency for SAW with high transmission in a narrow bandwidth. The system presents perspectives for the design of high quality-factor phononic excitation for optomechanic devices and phonon circuits based on SAW manipulation.
\end{abstract}

\section{Introduction}

Phononic crystals (PCs) are highly dispersive materials for elastic-acoustic waves, generally made of periodic distribution of solid materials and/or fluids with different acoustic wave velocities [1,2]. They can exhibit frequency band gaps (BG) where acoustic-elastic waves' propagation is prohibited which opens the way toward engineering PC based devices for filtering [3-5], waveguiding [6-8], sensing [9,10], acoustic focusing $[11,12]$, lensing $[13,14]$ and topological phononics $[15,16]$. Shortly after the PC were proposed, the phononic community turned their interest into acoustic metamaterials (AM) as they perform 
unusual physical properties such as negative effective mass density and/or bulk modulus or compressibility [17-20]. These properties paved the way towards the design of artificial systems capable of breaking conventional physical limitations and achieving remarkable acoustic wave manipulation such as sound insulation [21], sub-wavelength focusing [22,23], and acoustic clocking [24]. Usually, an AM contains periodic distribution of resonators basically made of soft material distributed on a stiff hosting media in such a way that the local resonances of the resonators allow the creation of sub-wavelength BGs. At the BG's frequency range, the wavelengths in the hosting media are by several orders of magnitude larger than the periodicity of the system.

As interest grows on the design of PC and AM for efficient control of acoustic/elastic waves, many studies have dealt with solid structures based on plates or membranes as they offer efficient waveguiding for elastic waves, with low loss and weak modes' conversion for large wavelengths compared to the plate's thickness. Besides plates with periodic inclusions $[25,26]$, numerous works investigated the Lamb waves dispersion on a thin plate decorated with homogenous pillars on one of its free surface. Pennec et al. [7,27] and $\mathrm{Wu}$ et al. [28,29] introduced a PC made of a plate with pillars which can display a lowfrequency BG occurring at wavelengths larger than the distance between pillars. The BG is created by the local resonance of the pillars which couples with the Lamb waves at low frequency. This property was used to achieve waveguiding by creating a defect inside the system. Wu et al. [28] conducted a series of laser ultrasonic experiments to evidence the BG presence on the PC plate and found good agreement between theoretical and experimental results. Another system was proposed using soft material pillars made of silicone on a thin aluminum membrane [20,30,31]. It was demonstrated theoretically and experimentally that the structure can produce tunable resonance BG for wavelengths larger than the periodicity of the structure [32]. Lamb wave manipulation and waveguiding was also demonstrated experimentally in a stubbed plate in the sonic regime $[33,34]$. Recently, the scattering of Lamb waves by a single or a line of pillars was studied with an emphasis on the determination of amplitude and phase of the secondary waves emitted by the resonators constituted by the pillars [35]. Exerting a force of a given strength and phase on the pillars opened the way to an active control of transmission.

Deeper investigations on pillar based plate systems were conducted to take advantage of their special properties so as to achieve an efficient control of Lamb waves such as confinement and waveguiding. In fact, by introducing a point or a line defect either by removing pillars or replacing them by others with different elastic properties, elastic energy can be confined inside the defect and acoustic modes with zero or slow group velocity can be created inside the BG. Pennec et al. [27] demonstrated this feature by introducing a line defect via increasing the space between two rows of pillars inside the phononic dots. They also analyzed different waveguides constructed by either removing one row of pillars, changing their height or the material [7], and evidenced the elastic wave transmission and 
confinement through the line defect. Furthermore, another kind of structure was proposed with hollow pillars for taking advantage of the whispering gallery modes which can enhance the confinement of the local resonances [36]. The modes can be manipulated by filling the hollow parts with a liquid [37,38]. When a waveguide was created by changing the inner radii of a row of pillars, the elastic waves were efficiently guided inside the structure at frequencies inside the BG [36]. Wu et al. [29] have also shown the same properties for a stubbed plate PC by considering a bent waveguide and experimentally evidenced the elastic waveguiding. These innovative systems are promising for the design of acoustic devices intended for filtering, multiplexing and sensing in high frequency applications. Besides, the AM plate decorated with pillars showed also great potential in low frequency for the control of acoustic waves in the sonic regime. The sub-wavelength BG property can be used to construct a thin sonic insulator with high sound transmission losses [39]. Furthermore, by introducing a defect inside the system, acoustic energy can be confined into a spot where it can be electromechanically converted for the purpose of acoustic energy harvesting [40].

In the very-high-frequency regime, some works have been initiated testing the performance of PC to control surface acoustic waves (SAW) for the purpose of sensing and filtering applications. Besides PC with holes or finite inclusions on substrate [5, 8, 41, 42], PC made of pillars distributed over a surface of semi-infinite material has been proposed. Khelif et al. [43] investigated theoretically the dispersion of SAW in a two-dimensional array of cylindrical pillars and showed the possibility to open a BG thanks to the local resonance of individual pillars. It was also shown that a pillar based PC appears to be more suitable for the creation of SAW BG than holes or inclusions on the surface of the substrate. In fact, SAW interaction with structures often results in mode conversion which affects the transmitted signal. In the case of finite depth holes on substrate, leaky SAWs can be dominant and lead to evanescent modes which couple with bulk acoustic waves (BAW) in the substrate. This causes a quick decay of the transmitted wave amplitude with propagation distance. The phenomena can be depicted in the linear dispersion curves where the SAW-BAW coupling occurs at frequencies above the sound line. In the case of local resonance BG (LR BG) created by pillars, the couplings between the resonance modes and SAW occur below the sound line, which therefore causes less energy leakage into the bulk. Robillard et al. [44] have detected individual vibrations of cubic metallic pillars distributed in a two-dimensional lattice using an ultrafast laser pump and probe setup, through a photoelastic mechanism. Achaoui et al. [45] used optical measurements to demonstrate the arising of LR BG in which the acoustic energy is confined inside the pillars and the SAW transmission is reduced. Yudistira et al. [46] proposed a pillar-based hypersonic PC by which they evidenced both theoretically and experimentally the Bragg and LR BG. The SAW dispersion was also investigated experimentally in two-dimensional lattice of metallic nanodisks [47]. Very recently, Ash et al. [48] proposed a well-tailored PC system made of pillars in holes or what can be 
seen as ring holes capable of supporting local resonances which creates SAW BG. For civil engineering application, Brûlé et al. [49] presented a macro-scale metamaterial for seismic waves' manipulation. Later, Colombi [50] skillfully showed that a forest can be seen as a metamaterial where trees behave like resonators which strongly attenuate Rayleigh waves.

Regarding SAW control and waveguiding, Lethawe et al. [51] investigated the possibility of guiding the elastic wave energy through a chain of pillars on the surface of a solid substrate. at In the proposed PC structures for SAW control, although the process of introducing a defect inside the PC allows the creation of SAW modes inside the BG, the band width of the confined mode remains generally large and entirely closes the BG. This behavior restrains the possibility to design high quality factor (QF) SAW modes in these systems for the purpose of sensing applications for instance.

In this work we propose a geometry of pillared PC on a substrate in which each pillar is constituted by a periodic stack of alternating layers (Fig.1). The difference of this structure with respect to the previous cases is that the pillar behaves by itself like a one-dimensional (1D) PC, so it allows BGs that prohibit propagation along the pillar. Hence, one can design highly confined modes within a cavity or on the upper surface of the pillar or at its interface with the substrate. Therefore, among the local resonances of the pillars that interact with the SAW, some will be very confined inside the pillars with a very high QF. The purpose is to study the excitation of these modes and their coupling with SAW, and investigate the possibility to obtain the interaction in a very narrow bandwidth to produce either sharp SAW attenuation or very high transmission with almost no leakage into the bulk substrate. Additionally, we investigate some specific properties that could be observed and achieved for SAW such as Fano like resonances and an acoustic analog of electromagnetically induced transparency (EIT) with high transmission in a narrow bandwidth which, to the best of our knowledge, has never been realized before. The paper is organized as follows: Sec. II presents the phononic pillars based PC and discusses the SAW dispersion through the system. In Sec III, we conduct SAW transmission calculations upon rows of pillars to investigate their modes' coupling with SAW and discuss the main physical phenomena displayed by the system.

\section{SAW dispersion in the PC system with phononic pillars}

We consider a pillar constructed from an infinite phononic cylinder composed of successive layers of silicon ( $\mathrm{Si}$ ) and PMMA (a periodic beam) with the same thickness $h$ and diameter $d$ (Fig.1). For the chosen thickness $h=3.5 \mu \mathrm{m}$ and diameter $d=5 \mu \mathrm{m}$, the band structure (BS) calculation for wave propagation along the periodicity, i.e. $\boldsymbol{k}_{\mathrm{z}}$ direction in Fig.1, shows the existence of two BG denoted BG1 from 133.6 to 
$181 \mathrm{MHz}$ and BG2 from 251.5 to 292.4 MHz. All the theoretical calculations are performed using the finite elements method.

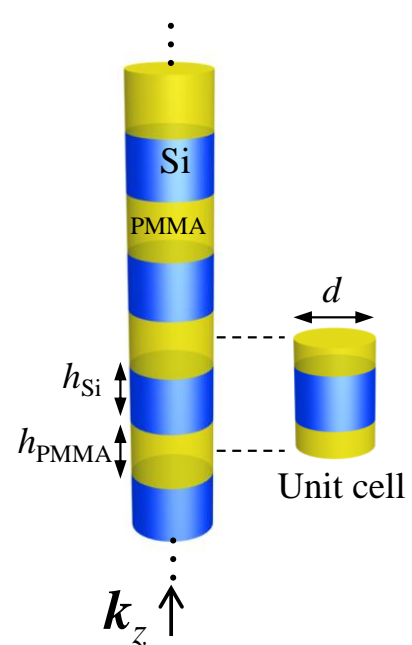

Phononic cylinder

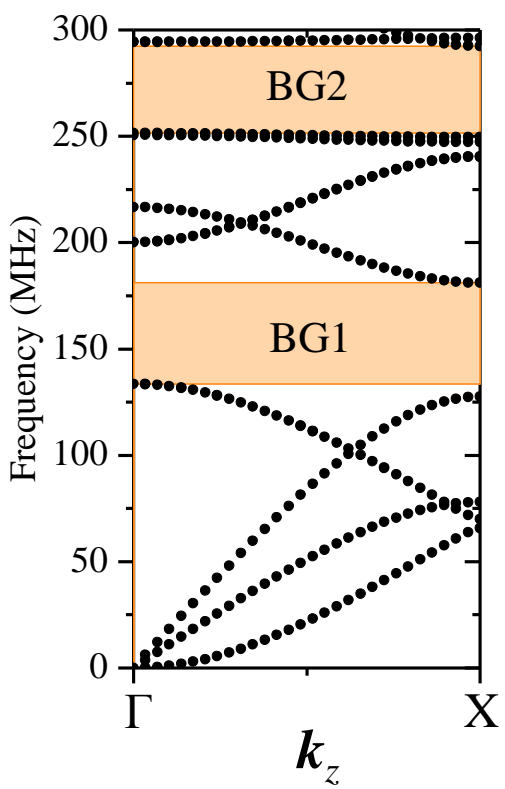

Fig.1. (Left) Phononic cylinder composed of alternating layers of silicon and PMMA. (Right) the band structure along the $\boldsymbol{k}_{\mathrm{z}}$ direction for $h=h_{\mathrm{Si}}=h_{\mathrm{PMMA}}=3.5 \mu \mathrm{m}$ and $d=5 \mu \mathrm{m}$.

Then, for SAW dispersion investigation, we consider a PC structure composed of a square distribution of phononic pillars on the surface of the thick silicon substrate (Fig.2(a)). Each pillar is constructed with 6.5 periods so that a silicon layer is on both of its ends. The periodicity of the PC, i.e. the distance between two pillars, is $a=6 \mu \mathrm{m}$ and a unit cell is considered with periodic boundary conditions (PBC) along the $x$ and $y$ directions to calculate the SAW BS presented in Fig.2(b). The X point is the Brillouin zone edge $\pi / a$ in the propagation direction $x$ for SAW. The region above the Si sound line, shaded in cyan, corresponds to the modes that either belong to the substrate or are leaky surface resonances which radiate into the silicon substrate. Below the Si sound line, the modes are localized at the surface of Si substrate and/or inside the pillars. However, among these modes, a few appear in the orange shaded regions which correspond to the phononic pillar band gaps BG1 and BG2. These modes are not only prohibited for propagation into the Si substrate, but they are localized in specific regions of the pillars as explained below. This is in contrast with the other SAW branches for which the displacement fields are spread all along the pillars. 
(a)

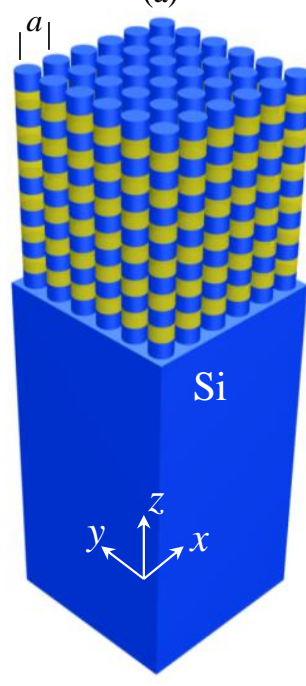

PC SAW system

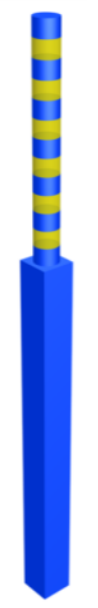

Unit cell with PBC

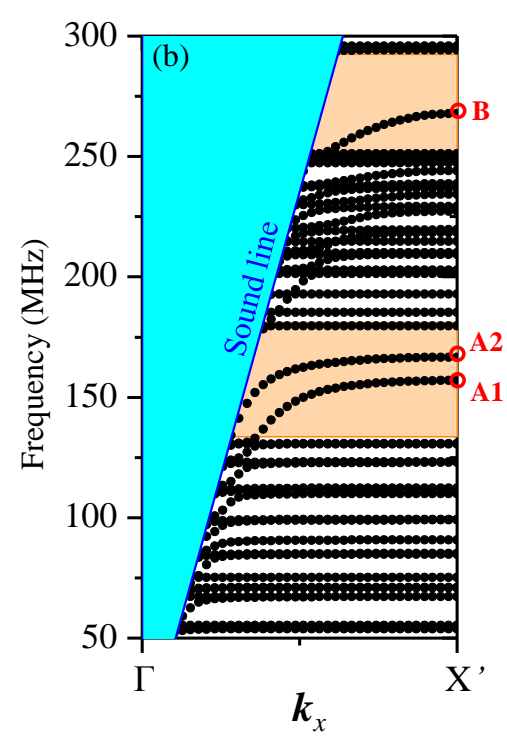

Fig.2. (a) PC structure made of a square array of phononic pillars on the Si substrate surface. (b) Band structure of the PC system for SAW propagation along the $x$ direction. (c) Total displacement field amplitude in the unit cell for modes denoted A1, A2 and B in the band structure

We observe in the BG regions that the system displays three particular resonance modes: two denoted A1 and A2 at 157 and 166.6 MHz respectively inside BG1, and one mode denoted B at 267.8 $\mathrm{MHz}$ inside BG2. The norm of total displacement field amplitude together with the shape of the deformation of the three modes plotted in Fig.2(c) shows that they are local resonance modes with almost zero group velocity near the $X^{\prime}$ point of the first Brillouin zone. Furthermore, the mechanical vibration is confined within the bottom of the pillar where the maximum of the displacement field amplitude is located in Si and PMMA layers close to the substrate surface. This amplitude quickly decays along the upper Si/PMMA layers of the pillar to have almost no vibration on the top layers. This behavior can be explained by the presence of the band gaps BG1 and BG2 (shaded orange regions) of the phononic pillar which prohibit any wave propagation along the whole pillar. Additionally, when having a close look into the mode's shape, modes A1 and A2 have flexural like vibrations of the pillar, mainly along $x$ and $z$ directions for mode $\mathrm{A} 1$ and along the $\mathrm{y}$ and $\mathrm{z}$ direction for the mode $\mathrm{A} 2$. The mechanical vibrational motion of mode $\mathrm{B}$ is mainly along the $z$ direction and corresponds to a compression like vibration where the two bottom PMMA layers are stretched and compressed along the $z$ direction. Besides, we point out that the flat modes located outside the band gaps BG1 and BG2 are resonance modes of the pillar where the mechanical vibration involves the whole pillar's layers. Such modes are, therefore, of less interest in this study. 
Afterwards, we investigate the phononic pillar behavior when introducing a geometrical defect. The latter is constructed by changing the thickness of the central Si layer (Fig.3(a)) denoted $\mathrm{W}_{\mathrm{Si}}$ in this study. The calculation is performed upon a finite phononic pillar where the central Si defect layer is stuck between three periods of PMMA/Si layers in such a way that the whole pillar has a Si layer on both of its ends. Furthermore, we fix the bottom surface of the pillar while keeping the upper surface free. When choosing for example a thickness of $\mathrm{W}_{\mathrm{Si}}=1.2 \mu \mathrm{m}$, five kinds of confined modes are depicted in the band gap BG1 and one mode exists in BG2. The investigation of the total displacement field amplitude (Fig.3(b)) of these modes leads us to classify them into two main families. The first one is for cavity modes denoted E, F1 and F2 where the elastic energy is confined within the close vicinity of the geometrical defect, more precisely on the central Si layer and adjacent PMMA layers. The cavity mode E is located at $143 \mathrm{MHz}$ and has a compressional ibrational motion shape in which the PMMA layers in contact with the thin Si central layer are stretched and compressed along the $\mathrm{z}$ direction. We call this kind of mode compressional cavity mode (E) in this study. The two other cavity modes F1 and F2 are located at the same frequency $(\sim 149.45 \mathrm{MHz})$ and have a similar mechanical vibration shape where the cavity undergoes flexural behavior along the Si thin central layer and the two adjacent PMMA layers. The difference between them lies in the fact that the flexural vibration occurs along the $x$ or $y$ direction as it can be deduced from Fig.3(b). The second family gathers three confined modes denoted A1, A2 and B where the elastic energy is mainly concentrated within the bottom layers of the phononic pillar. The upper PMMA/Si layers do not display almost any vibrational motion. The first two resonant modes A1 and A2 are located at the same frequency $(181 \mathrm{MHz})$ very close to the upper edge of BG1. They are similar to the ones observed in the PC system (Fig.2) as they display flexural vibrations, mainly along $x$ and $z$ directions for mode A1, and along $y$ and $z$ directions for the mode A2. The third mode B, located at $290.4 \mathrm{MHz}$ in BG2, is a resonance mode where the bottom PMMA/Si layers are stretched and compressed along $z$ direction. Its mechanical behavior is quite similar to the compressional mode B observed in the PC system at BG2 (Fig.2). The fact that the three modes A1, A2 and B are not exactly located in the same frequency as in the case of the PC system presented in Fig. 2 is explained by the nature of the boundary condition of the pillar's bottom surface. The cavity mode's calculations are performed with a fixed boundary in Fig. 3 while the pillar is actually in contact with an elastic Si substrate in the case of Fig.2.

Figure 3(c) shows the frequency dependence on the Si layer thickness $\mathrm{W}_{\mathrm{Si}}$ for the six localized modes where for the cavity modes, the black dots indicate the mode $\mathrm{E}$ while the blue triangles and red circular dots are for cavity modes F1 and F2 respectively which occur at the same frequency in the BG1. For the three other confined modes in the bottom layers of the phononic pillar, the red diamonds, the green open squares and the inverted purple triangles indicate the frequencies of modes A1, A2 and B respectively. The orange shaded regions cover the band gaps BG1 and BG2. One can deduce from the 
result that for each cavity mode E, F1 and F2, the frequency increases when lowering the thickness of the Si layer defect. This behavior can be expected knowing that the cavity modes tend to adapt to the cavity size so that their wavelength becomes smaller when decreasing $\mathrm{W}_{\mathrm{Si}}$. Meanwhile, the frequency of the confined modes $\mathrm{A} 1, \mathrm{~A} 2$ and $\mathrm{B}$ remains almost unchanged when changing $\mathrm{W}_{\mathrm{Si}}$ which is expected knowing that the elastic strain energy is confined within the bottom layers far from the cavity defect.

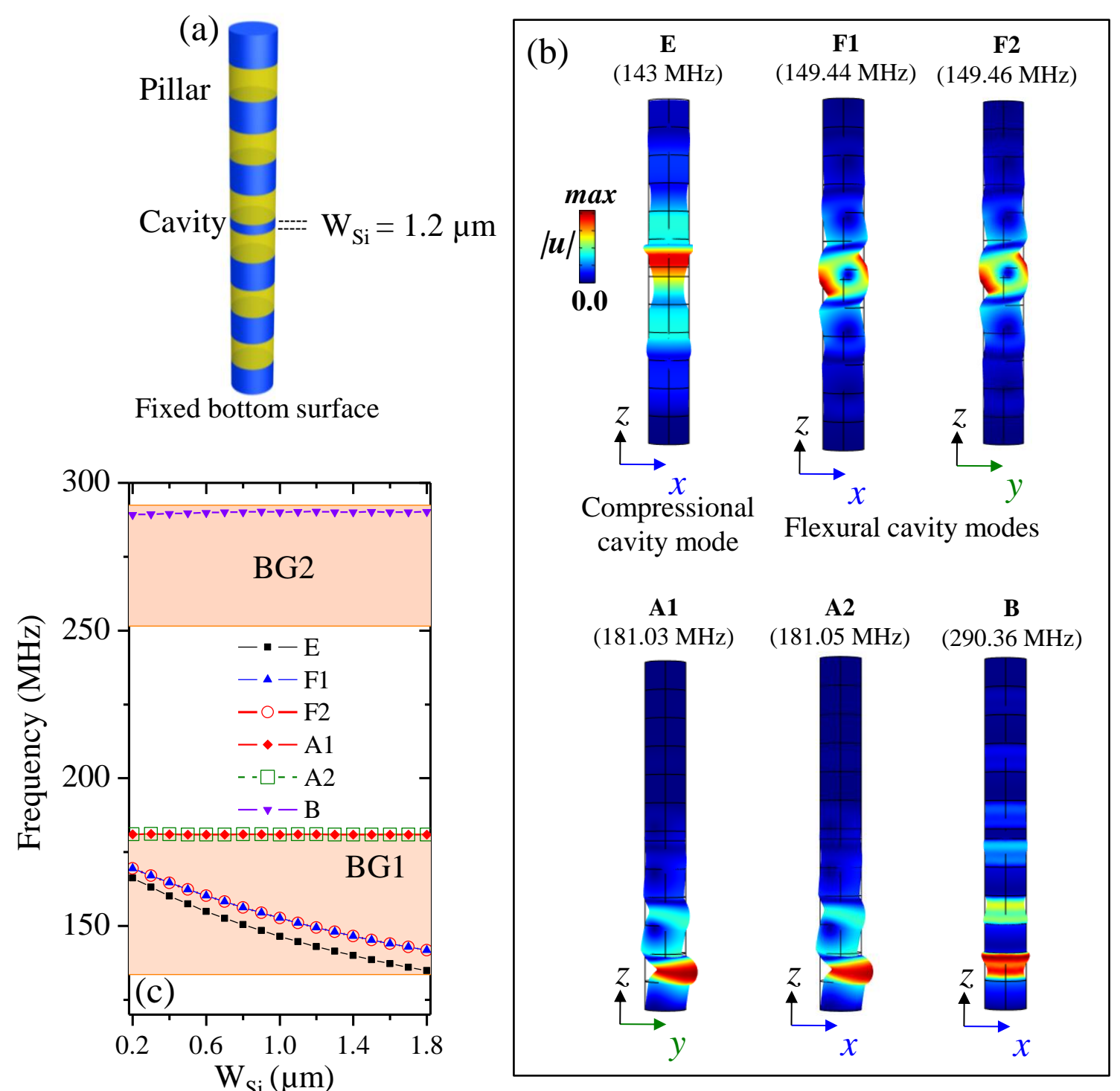

Fig.3. (a) Phononic pillar where the structural defect is constructed by changing the thickness $\mathrm{W}_{\mathrm{Si}}$ of the central $\mathrm{Si}$ layer. The bottom surface of the pillar is fixed. (b) Total displacement field amplitude for the confined modes in the phononic pillar for $\mathrm{W}_{\mathrm{Si}}=1.2 \mu \mathrm{m}$. (c) The confined modes frequency shift as function of $\mathrm{W}_{\mathrm{Si}}$.

In the rest of this section, we investigate the SAW interaction with the confined modes of the phononic pillars distributed in a square lattice form on the surface of the Si substrate with $6 \mu \mathrm{m}$ periodicity. 
Figure 4(a) shows the BS calculation for SAW dispersion and interaction with the pillars where the thickness of the central $\mathrm{Si}$ layer is $\mathrm{W}_{\mathrm{Si}}=1.2 \mu \mathrm{m}$. In the frequency band of the first band gap BG1, we can depict five local resonance modes which couple with SAW. An enlargement of the BG region is plotted in Fig.4(b) to see more clearly the modes coupling. We also display in Fig.4(c) the displacement field profiles of these modes to identify their mechanical motion. When analyzing the displacement fields, we can first clearly identify the flexural confined modes A1 and A2 and the compressional mode B which correspond to the same modes depicted in Fig.2(b). Second, the BS of Fig.4(b) displays three additional modes denoted E, F1 and F2 in the BG1 which correspond to the cavity modes of the phononic pillar identified in Fig.3(b). One can expect that coupling between SAW and the pillars will lead to the excitation of the cavity modes which can be seen as flat modes in the band structure where their group velocity is zero. Furthermore, in the $\mathrm{X}^{\prime}$ point, the compressional cavity modes $\mathrm{E}$ is located at $143 \mathrm{MHz}$ while modes F1 and F2 are located at the same frequency $149.2 \mathrm{MHz}$ which are almost the same frequencies as in the case of the isolated pillar presented in Fig.3. 


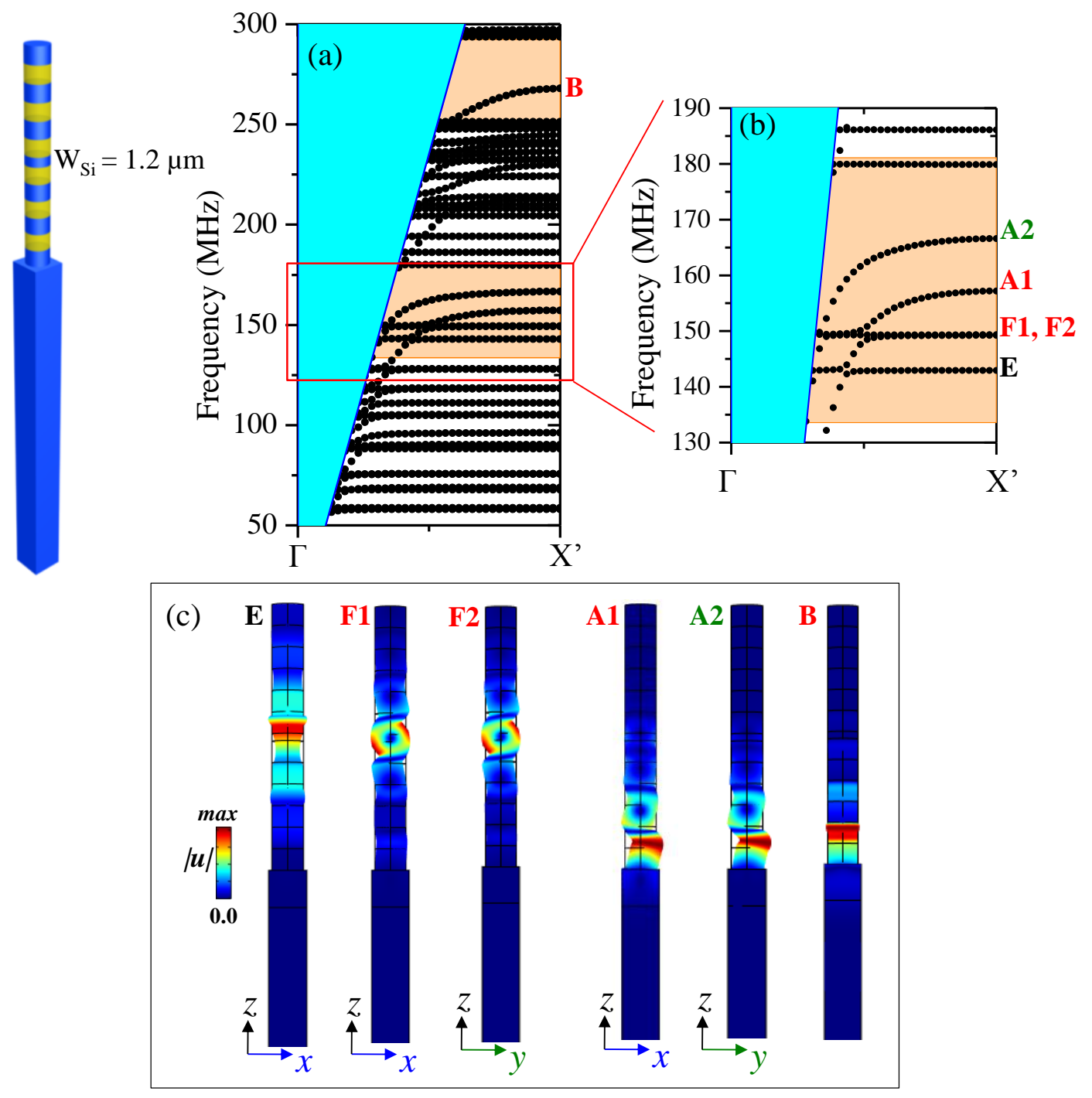

Fig.4. (a) Band structure for PC system composed of phononic pillars with thickness of the central Si layer $\mathrm{W}_{\mathrm{Si}}=1.2 \mu \mathrm{m}$. (b) Enlargement of the band gap region BG1. (c) Total displacement field amplitude $|\boldsymbol{u}|$ for the confined modes inside the band gaps at $\mathrm{X}^{\prime}$ point.

\section{SAW transmission through a row of phononic pillars}

To investigate deeply the SAW interaction with the phononic pillars and understand the coupling mechanisms between the modes of interest, we perform transmission calculations on one row of pillars as it is schematically described in Fig.5(a). We use the same finite phononic pillar as displayed in Fig.2 with 6.5 periods of Si/PMMA layers and both upper and bottom ends of the pillar are made of Si layer. The model used for the transmission calculation is presented in Fig.5(b) where we used IDT lines footprints to excite the surface wave at the central frequency $154 \mathrm{MHz}$ of the band gap BG1. We also consider one unit cell along the $y$ direction with PBC to describe the periodicity of the system. The period between the two adjacent pillars is $a=6 \mu \mathrm{m}$ and perfectly matched layers (PML) are used to model the infinite wave 
propagation and avoid reflections. Knowing that SAW have displacement field components along the propagation direction $x$ and the $z$ direction normal to the surface, we evaluate the transmission by averaging the amplitude of the out-of-plane displacement component $u_{\mathrm{z}}$ along the output line indicated in Fig.5(b).

The transmission result plotted in Fig.5(c) shows SAW attenuation in the BG1 region (shaded region) where the amplitude of the $u_{\mathrm{z}}$ component drops by $60 \%$ at $161 \mathrm{MHz}$ (denoted $\mathrm{A}$ in the figure). At this frequency, we plot in Fig.5(d) the total displacement field in the phononic pillar as well as the $u_{\mathrm{z}}$ component in the substrate. One can deduce that the SAW attenuation is caused by the excitation of a wideband localized mode having a vibrational motion quite similar to the confined mode denoted A1 depicted in the BS (Fig.2(b)) for the PC system. The pillar displays flexural vibration at its bottom in the PMMA/Si layers in the vicinity of the substrate's surface and the mode couples with SAW causing the mode's conversion and leakage into the bulk substrate. Furthermore, when looking closely into the mechanical vibration amplitudes, we can notice that at the minimum transmission frequency, the maximum displacement amplitude in the pillar is 3.61 times the $u_{\mathrm{z}}$ amplitude of SAW.

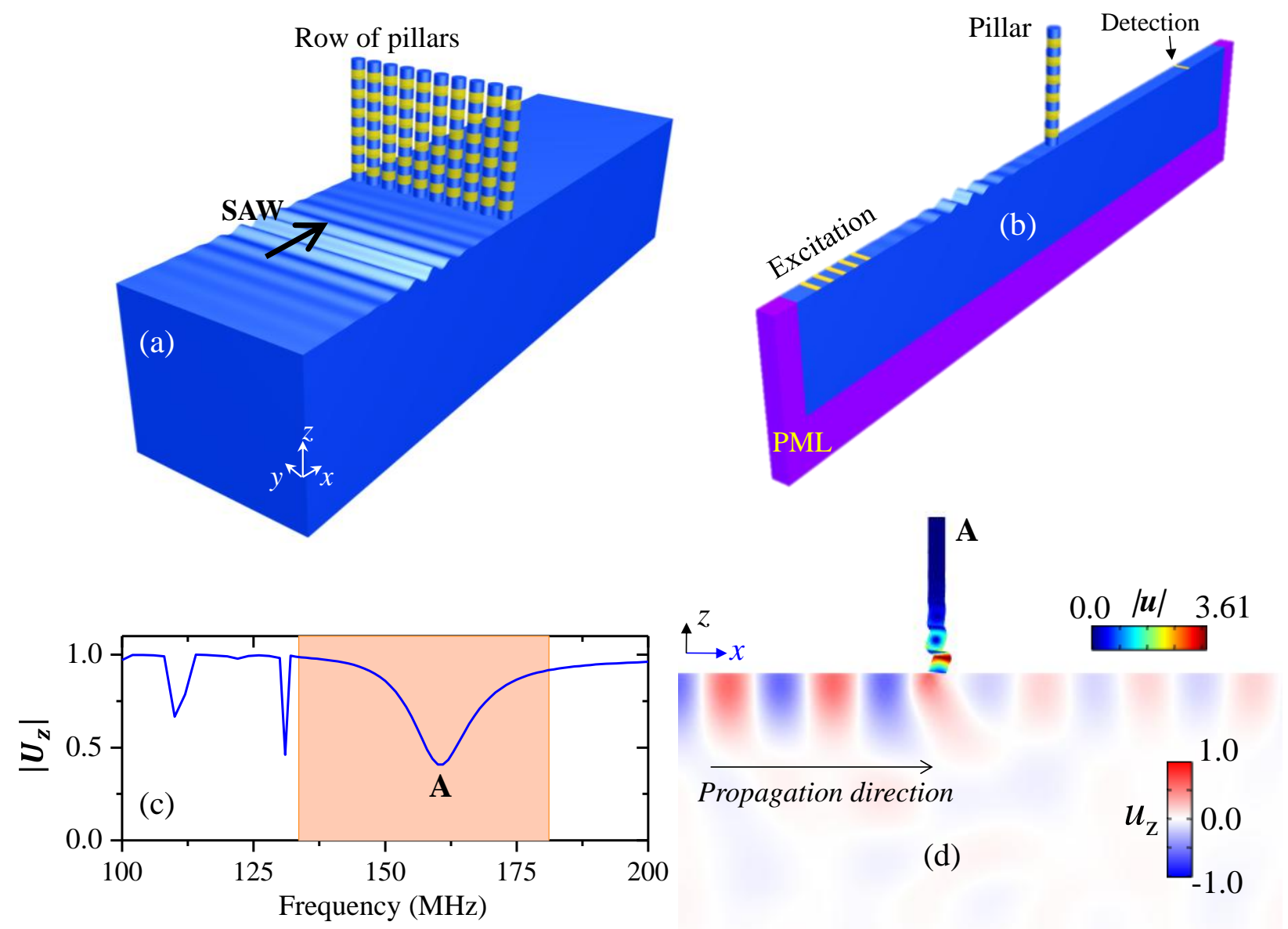

Fig.5.(a) Schematic view for the transmission through one row of PC pillars. (b) The model used for FEM calculation with PBC and PML. (c) Transmission results : normalized out-of-plane displacement's amplitude Uz. 
The shaded region is for the phononic BG of the pillar. (d) Total displacement field in the pillar as well as the $u_{\mathrm{z}}$ component in the Si substrate for SAW.

In the following study, we calculate the transmission of SAW through a row of phononic pillars with defect for different thicknesses $\mathrm{W}_{\mathrm{Si}}$ of the Si central layer. Figure 6 shows the results where $\mathrm{W}_{\mathrm{Si}}$ is changed from $1.2 \mu \mathrm{m}$ to $0.5 \mu \mathrm{m}$. In addition to the large attenuation band A depicted in the case of a phononic pillar without defect and located at $161 \mathrm{MHz}$, we notice the presence of two sharp transmission dips denoted $\mathrm{E}$ and $\mathrm{F}$ in the panel $\mathrm{W}_{\mathrm{Si}}=1.2 \mu \mathrm{m}$. To figure out the vibrational nature of these two modes, we display in Fig.7(a) and 7(b) the associated displacement fields where we plot the norm of the total displacement field amplitude in the pillar while we plot the out-of-plane component $u_{\mathrm{z}}$ in the substrate. We can easily deduce that mode $\mathrm{E}$ is the compressional cavity mode at $143.1 \mathrm{MHz}$ while mode $\mathrm{F}$ at $149.55 \mathrm{~Hz}$ is the flexural cavity mode observed beforehand in Fig.4. Both cavity modes can be excited by SAW interaction with the pillar.

Meanwhile, upon decreasing the Si layer thickness $\mathrm{W}_{\mathrm{Si}}$, the frequencies of modes $\mathrm{E}$ and $\mathrm{F}$ increase to meet the wide band attenuation created by mode $\mathrm{A}$. In this region, in the case of $\mathrm{W}_{\mathrm{Si}}=0.9 \mu \mathrm{m}$, the mode F displays a well distinguished asymmetric peak/dip feature in the transmission. When the Si thickness is chosen between $0.5 \mu \mathrm{m}$ and $0.6 \mu \mathrm{m}$, the frequency of mode F falls into the SAW attenuation band defined by the confined mode A. The transmission curve displays an asymmetric peak known as Fano-like resonance in the form of high SAW transmission with a very narrow band. The origin of this Fano-like [53] behavior comes from a strong coupling between the cavity mode $\mathrm{F}$ and the localized wide band mode A. These two modes have the same mechanical vibrational motion as they display flexural behavior of the pillar, so their strong coupling is expected. Although the mechanism is different, Nardi et al. $[54,55]$ have evidenced a Fano resonance created by the coupling between surface modes and BAW in PC.

To investigate deeply the Fano-like peak resonance, we look into the mechanical behavior of the system in the case of $\mathrm{W}_{\mathrm{Si}}=0.7 \mu \mathrm{m}$ at the low transmission frequency denoted $\mathrm{F}(158.05 \mathrm{MHz})$ and high transmission peak indicated by $\mathrm{D}$ at $158.55 \mathrm{MHz}$ in the right panel of Fig.6. The displacement fields plots at these two frequencies are displayed in Fig.7(c) and 7(d). One can easily distinguish the flexural cavity mode excitation by SAW. At these very close frequencies, we can have low SAW transmission of $41 \%$ at $158.05 \mathrm{MHz}(\mathrm{F})$ and very high SAW transmission of $98.7 \%$ at $158.55 \mathrm{MHz}$ at the peak D. Furthermore, we observe that the vibration amplitude inside the cavity is more than 90 times higher than the SAW amplitude for the peak D. Besides, when looking closely into the case $\mathrm{W}_{\mathrm{Si}}=0.6 \mu \mathrm{m}$, we observe an analog of the EIT [56,57] where a narrow band with high SAW transmission of $97.5 \%$ is depicted at the peak 160.57MHz. To the best of our knowledge, this behavior has never been achieved for SAW. In plasmonic crystals for instance, the EIT-like behavior is demonstrated by the coupling between localized surface 
states (known as Tamm states) and the defect plasmonic state [58]. Meanwhile, in all the transmission cases displayed in Fig.6, we also observe the presence of the sharp dip denoted E corresponding to the compressional cavity mode which does not couple with the wide band mode A. Hence, the cavity mode undergoes high attenuation of SAW without displaying particular behavior compared to the cavity mode F.

It is important to indicate that although we have dealt with one row of pillars while the BS is for the case of infinite PC in the previous section, the BS gives a very close idea about the resonance modes existence in the pillars and their interaction with SAW. The involved local resonance mechanism in the interaction makes the dispersion almost independent from the periodicity of the system.

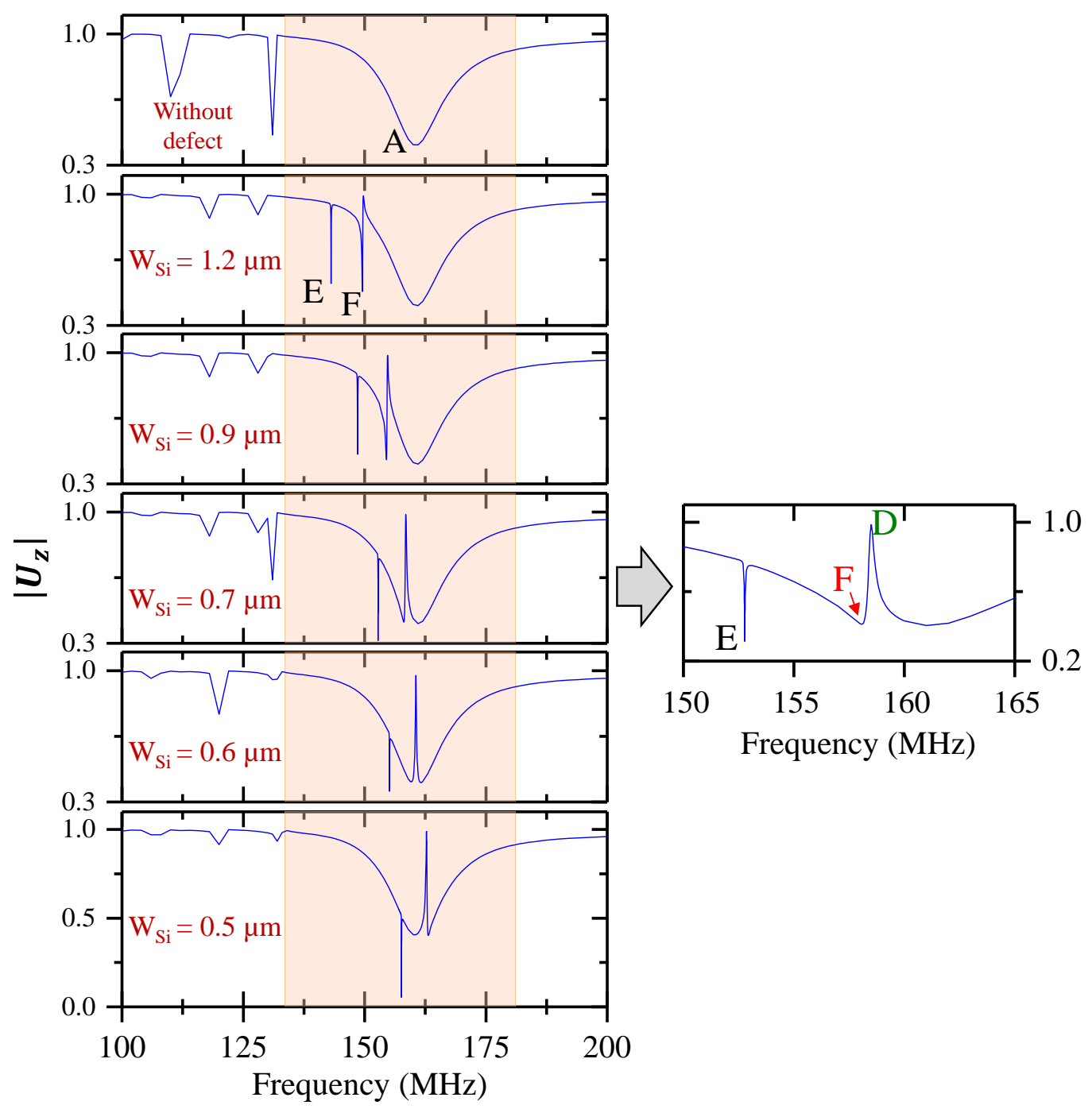

Fig.6. SAW transmission through a row of PC pillar for central Si layer thickness from $\mathrm{W}_{\mathrm{Si}}=1.2 \mu \mathrm{m}$ to $0.5 \mu \mathrm{m}$. The right panel is an enlargement in the region of interest for the case of $\mathrm{W}_{\mathrm{Si}}=0.7 \mu \mathrm{m}$ 


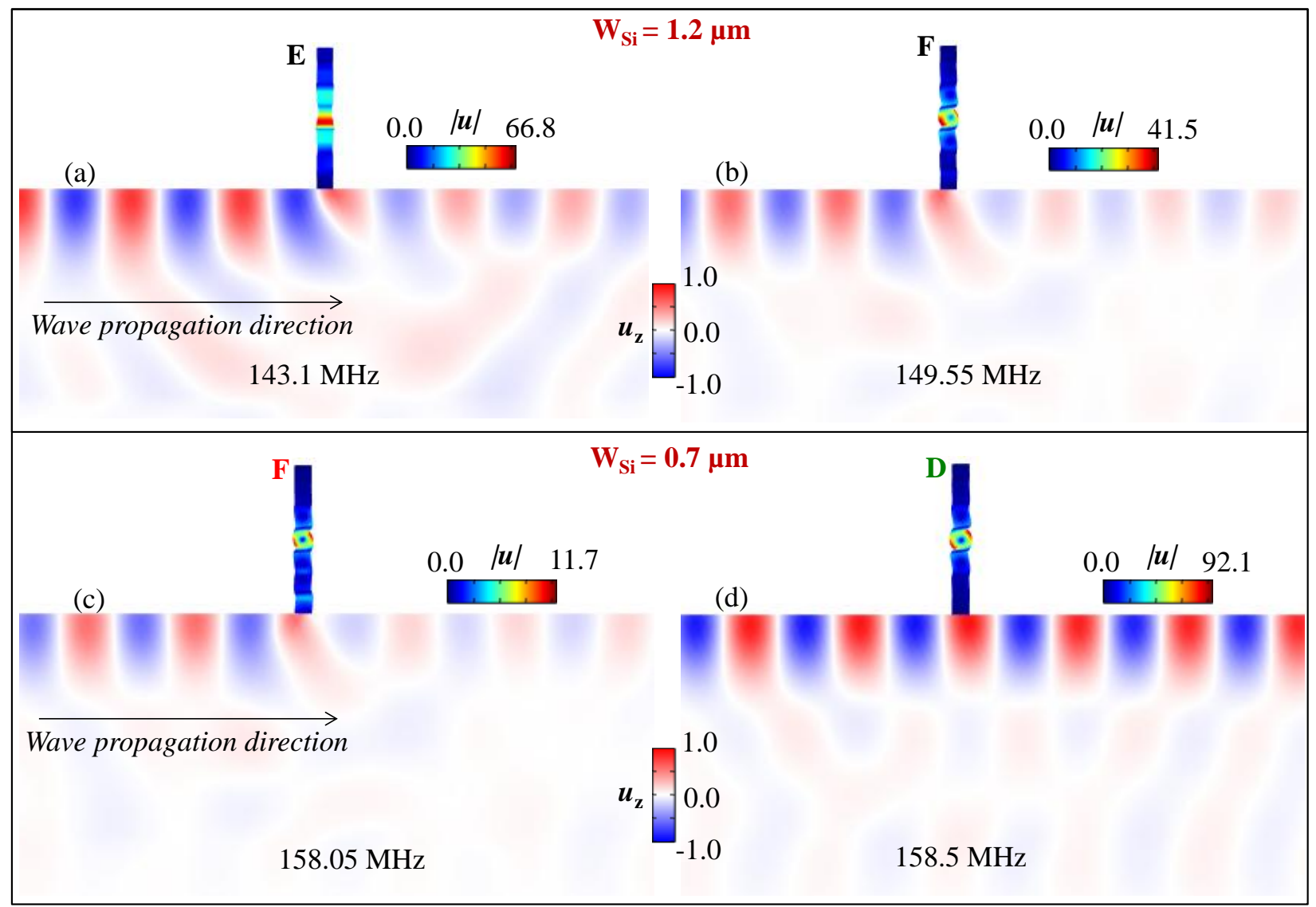

Fig.7. Total displacement field in the pillar as well as the $u_{\mathrm{z}}$ component in the Si substrate for SAW at the frequencies of the modes E (a), F (b)-(c), and D (d) indicated in Fig.6 for Wsi=1.2 $\mu \mathrm{m}$ and $0.7 \mu \mathrm{m}$.

Finally, to complete this study, we compute the SAW transmission over three rows of PC pillars spaced with $18 \mu \mathrm{m}$ as it is schematically shown in Fig.8(a). This value is chosen to avoid interaction between the pillars. All the pillars have their central Si layer thickness fixed to $\mathrm{W}_{\mathrm{Si}}=0.5 \mu \mathrm{m}$. The result in fig.8(b) shows almost the same behavior as the one observed for the single row of pillars (Fig.6) with the difference that the SAW attenuation observed in the BG region is higher in the case of three rows of pillars as the minimum transmission reaches $5.7 \%$ instead of $40.8 \%$ in the case of the single row of pillars. This low transmission can be explained by the excitation of the confined mode $\mathrm{A}$ in the three rows of pillars which have combined effects to increase the attenuation of SAW. Besides, high SAW transmission of about $87 \%$ is observed at the peak frequency $162.77 \mathrm{MHz}$ in a very narrow band in between two attenuation bands with transmission coefficients lower than 6\%, which proves the feasibility of acoustically induced transparency for SAW. 


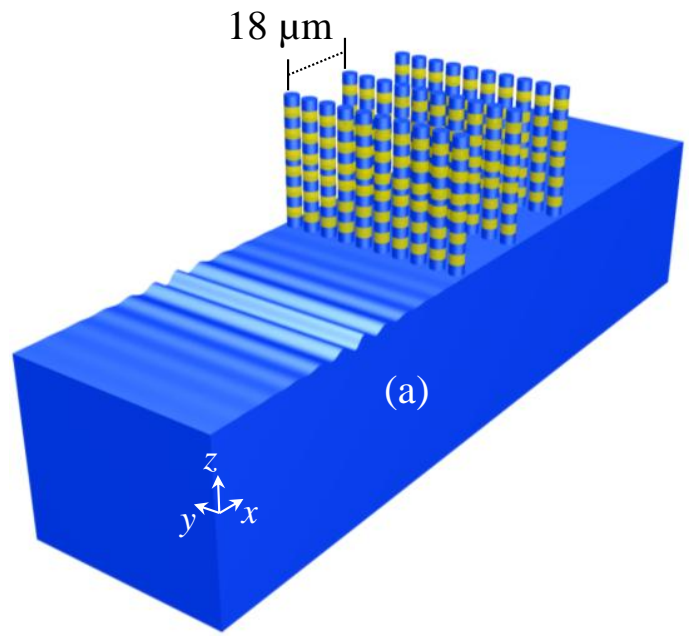

SAW
(c)

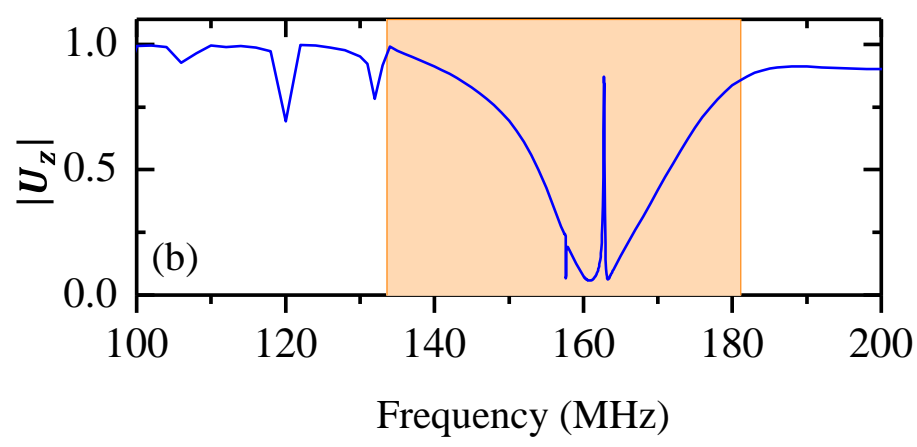

$0.0 \quad|u| \quad 92.9$

162.77 MHz

Fig.8. (a) Schematic view for the transmission through three rows of PC pillars spaced by $18 \mu \mathrm{m}$ in the $x$ direction.

(b) The SAW transmission result : normalized out-of-plane displacement's amplitude Uz. The shaded region is for the BG1 of the phononic pillar. (c) Total displacement field in the pillars as well as the $u_{\mathrm{z}}$ component in the $\mathrm{Si}$ substrate for SAW.

\section{Conclusion}

We investigate the band structure and transmission behavior of SAW in the presence of an array of pillars constituted by a periodic multilayer, possibly containing a cavity. Although the practical fabrication of such high aspect ratio pillars may be a technological challenge, it seems still feasible. The Si layer growth can be achieved from the $\mathrm{Cu} / \mathrm{Si}$ solution [59] or using $\mathrm{Si}$ layer deposition by gas-source molecular beam epitaxy for instance where thicknesses of few micro-meters can be reached [60]. Meanwhile, the PMMA layer can simply be deposited by the spin-coating process. Using these techniques, a multilayered system can be constructed, from which the pillars can be carved using the dry etching process.

We show that the phononic BG of the pillar undergoes the creation of resonance modes where the elastic energy is confined within the first phononic unit cells of the pillar in contact with the substrate surface, with no propagation through the whole pillar. These modes interact with SAW and cause a decay 
of the SAW amplitude in a relatively wide band. Furthermore, when introducing a defect into the multilayer phononic pillar, well confined cavity modes can be created which can couple with localized modes in the bottom of the pillar in contact with the substrate. The interaction can give rise to Fano like resonance behavior as well as an acoustic analog of EIT for SAW. The phononic pillar based system can be then envisaged as a solution for efficient manipulation of SAW with low leakage into the substrate. It opens perspectives to present an approach for SAW based devices design for high performance sensing. Besides, high QF confinement of mechanical modes is of the essence for optomechanic applications. With a phononic pillar based system, it would be for instance possible to excite a high QF phonon inside a welltailored optical cavity which can then couple with external photons. Very recently, Anguiano et al. [61] and Lamberti et al. [62] designed a composite GaAs/AlAs micopillar cavity capable of confining both phonons and photons with a strong enhancement of acousto-optical interaction. The concept opens promising perspectives to observe quantum optomechanic phenomena and also for the design of optomechanic based devices which could integrate microchips, for biosensing for instance with high sensitivity down to single molecule detection. Besides the cavity modes, another type of localized modes in the multilayer pillars may be those associated with their upper surface, as we investigated them in a very recent work (using ridges instead of pillars [63]). In the system studied in this paper, such modes can appear in the BG of the multilayer when changing the thickness or composition of the upper layer in the pillar. These modes can also interact with the interface modes and give rise to similar phenomena such as Fano and EIT resonances discussed above. Finally, one can imagine the possibility of interacting multiresonances when the phononic pillar contains two cavities or supports both surface and cavity modes. The frequencies of these modes can be fully controlled by the relative positions, thicknesses and elastic properties of the corresponding layers.

\section{Acknowledgments}

This work was supported by Université de Lorraine and the authors would like to thank Dr. E.H. El Boudouti for several fruitful discussions.

\section{References}

[1] M. S. Kushwaha, P. Halevi, L. Dobrzynski, and B. Djafari-Rouhani, Acoustic Band Structure Of Periodic Elastic Composites, Phys. Rev. Lett. 71, 2022 (1993).

[2] M. Sigalas and E. N. Economou, Band Structure Of Elastic Waves In Two Dimensional Systems, Solid State Communications, 86, 141 (1993).

[3] M. Kafesaki, M. M. Sigalas, and N. Garcia, Frequency Modulation in the Transmittivity of Wave Guides in Elastic-Wave Band-Gap Materials, Phys. Rev. Lett. 85, 4044 (2000). 
[4] C. Qiu, Z. Liu, J. Mei, and J. Shi, Mode-selecting acoustic filter by using resonant tunneling of twodimensional double phononic crystals, Appl. Phys. Lett. 87, 104101 (2005).

[5] T. T.Wu, L. C.Wu, and Z. G. Huang, Frequency band-gap measurement of two-dimensional air/silicon phononic crystals using layered slanted finger interdigital transducers, J. Appl. Phys. 97, 094916 (2005).

[6] J. O. Vasseur, P. A. Deymier, B. Djafari-Rouhani, Y. Pennec, and A-C. Hladky-Hennion. Absolute forbidden bands and waveguiding in two-dimensional phononic crystal plates, Phys. Rev. B 77, 085415 (2008)

[7] Y. Pennec, B. Djafari Rouhani, H. Larabi, A. Akjouj, J. N. Gillet, J. O. Vasseur, and G. Thabet, Phonon transport and waveguiding in a phononic crystal made up of cylindrical dots on a thin homogeneous plate, Phys. Rev. B 80, 144302 ( 2009)

[8] J.-H. Sun and T.-T. Wu, Propagation of surface acoustic waves through sharply bent two-dimensional phononic crystal waveguides using a finite-difference time-domain method, Phys. Rev. B 74, 174305 (2006)

[9] Y. Pennec, B. Djafari-Rouhani, J. O. Vasseur, A. Khelif, P. A. Deymier, Tunable filtering and demultiplexing in phononic crystals with hollow cylinders, Phys. Rev. E 69, 046608 (2004)

[10] S. Amoudache, R. Moiseyenko, Y. Pennec, B. Djafari-Rouhani, A. Khater, R. Lucklum, and R. Tigrine, Optical and acoustic sensing using Fano-like resonances in dual phononic and photonic crystal plate, J. Appl. Phys. 119, 114502 (2016)

[11] T.-T. Wu, Y.-T. Chen, J.-H. Sun, Sz.-C. Steven Lin, and T. J. Huang, Focusing of the lowest antisymmetric Lamb wave in a gradient-index phononic crystal plate, Appl. Phys. Lett. 98, 171911 (2011) [12] J. Zhao, B. Bonello, L. Becerra, O. Boyko, and R. Marchal. Focusing of Rayleigh waves with gradient-index phononic crystals, Appl. Phys. Lett. 108, 221905 (2016)

[13] J. Zhao, B. Bonello, R. Marchal, and O. Boyko, Beam path and focusing of flexural Lamb waves within phononic crystal-based acoustic lenses, New J. Phys. 16, 063031 (2014).

[14] S.-C. S. Lin, T. J. Huang, J.-H. Sun, and T.-T.Wu, Gradient-index phononic crystals, Phys. Rev. B 79, 094302 (2009).

[15] Yang, Z.; Gao, F.; Shi, X.; Lin, X.; Gao, Z.; Chong, Y.; Zhang, B. Topological Acoustics, Phys. Rev. Lett, 114, 114301 (2015)

[16] C. He, X. Ni, H. Ge, X.-C. Sun, Y.-B. Chen, M.-H. Lu, X.-P. Liu, and Y.-F. Chen, Acoustic topological insulator and robust one-way sound transport, Nature Physics 12, 1124 (2016)

[17] Z. Liu, X. Zhang, Y. Mao, Y. Y. Zhu, Zh. Yang, C. T. Chan, and Ping Sheng. Locally resonant sonic materials, Science 289, 1734 (2000)

[18] J. Li and C. T. Chan, Double-negative acoustic metamaterial, Phys. Rev. E 70, 055602 (R) (2004) 
[19] Y. Ding, Z. Liu, C. Qiu, and J. Shi, Metamaterial with Simultaneously Negative Bulk Modulus and Mass Density, Phys. Rev. Lett. 99, 093904 (2007)

[20] M. Oudich, B. Djafari-Rouhani, Y. Pennec, M. B. Assouar, and B. Bonello, Negative effective mass density of acoustic metamaterial plate decorated with low frequency resonant pillars, J. Appl. Phys. 116, 184504 (2014).

[21] J. Mei, G. Ma, M. Yang, Z. Yang, W. Wen, and Ping Sheng, Dark acoustic metamaterials as super absorbers for low-frequency sound, Nat. Comm. 3, 756 (2012)

[22] X. Zhou, M. B. Assouar, and M. Oudich. Subwavelength acoustic focusing by surface-waveresonance enhanced transmission in doubly negative acoustic metamaterials. J. Appl. Phy. 116, 194501 (2014)

[23] X. Zhou, M. B. Assouar, and M. Oudich, Acoustic superfocusing by solid phononic crystals, Appl. Phys. Lett. 105, 233506 (2014)

[24] M. Farhat, S. Enoch, S. Guenneau, and A. B. Movchan, Broadband Cylindrical Acoustic Cloak for Linear Surface Waves in a Fluid, Phys. Rev. Lett. 101, 134501 (2008)

[25] A. Khelif, B. Aoubiza, S. Mohammadi, A. Adibi, and V. Laude, Complete band gaps in twodimensional phononic crystal slabs, Phys. Rev. E 74, 046610 (2006)

[26] J. O. Vasseur, A.-C. Hladky-Hennion, B. Djafari-Rouhani, F. Duval, B. Dubus, Y. Pennec, and P. A. Deymier, Waveguiding in two-dimensional piezoelectric phononic crystal plates, Appl. Phys. Lett. 101, 114904 (2007)

[27] Y. Pennec, B. Djafari-Rouhani, H. Larabi, J. O. Vasseur, and A. C. Hladky-Hennion, Low-frequency gaps in a phononic crystal constituted of cylindrical dots deposited on a thin homogeneous plate, Phys. Rev. B 78, 104105 (2008)

[28] T.-T. Wu, Z.-G. Huang, T.-C. Tsai, and T.-C. Wu, Evidence of complete band gap and resonances in a plate with periodic stubbed surface, Appl. Phys. Lett. 93, 111902 (2008).

[29] T.-C. Wu, T.-T. Wu, and J.-C. Hsu, Waveguiding and frequency selection of Lamb waves in a plate with a periodic stubbed surface, Phys. Rev. B 79, 104306 (2009)

[30] M. Oudich, Y. Li, B. M. Assouar, and Z. Hou, A sonic band gap based on the locally resonant phononic plates with stubs, New J. Phys. 12, 083049 (2010)

[31] M. B Assouar, and M Oudich, Enlargement of a locally resonant sonic band gap by using doublesides stubbed phononic plates, Appl. Phys. Lett. 100, 123506 (2012)

[32] M. Oudich, M. Senesi, M. B. Assouar, M. Ruzzene, J.-H. Sun, B. Vincent, Z. Hou, and T.-T. Wu, Experimental evidence of locally resonant sonic band gap in two-dimensional phononic stubbed plates, Phys. Rev. B 84, 165136 (2011) 
[33] M. B. Assouar, M. Senesi, M. Oudich, M. Ruzzene, and Z. Hou, Broadband plate-type acoustic metamaterial for low-frequency sound attenuation, Appl. Phys. Lett. 101, 173505 (2012)

[34] P. Celli and S. Gonella, Manipulating waves with LEGO $^{\circledR}$ bricks: A versatile experimental platform for metamaterial architectures, Appl. Phys. Lett 107, 081901 (2015)

[35] Y. Jin, B. Bonello, R.P. Moiseyenko, Y. Pennec, O. Boyko and B. Djafari-Rouhani, Pillar-type acoustic metasurface, Phys. Rev. B 96, 104311(2017)

[36] Y. Jin, N. Fernez, Y. Pennec, B. Bonello, R. P. Moiseyenko, S. Hémon, Y. Pan, and B. DjafariRouhani, Tunable waveguide and cavity in a phononic crystal plate by controlling whispering-gallery modes in hollow pillars, Phys. Rev. B 93, 054109 (2016)

[37] Y. Jin, E. H. EI Boudouti, Y. Pennec, and B. Djafari-Rouhani, Phononic crystal plate with hollow pillars connected by thin bars. J. Phys. D: Appl. Phys. 50, 425304 (2017)

[38] Y. Jin, Y. Pennec, Y. Pan and B. Djafari-Rouhani, Phononic Crystal plate with hollow pillars actively controlled by fluid filling, Crystals 6, 64 (2016)

[39] M. Oudich, X. Zhou, and M. B. Assouar, General analytical approach for sound transmission loss analysis through a thick metamaterial plate, J. Appl. Phys. 116, 193509 (2014)

[40] M. Oudich, and Y. Li, Tunable sub-wavelength acoustic energy harvesting with a metamaterial plate, J. Phys. D: Appl. Phys. 50, 315104 (2017)

[41] T. T. Wu, Z. G. Huang, and S. Lin, Surface and bulk acoustic waves in two-dimensional phononic crystal consisting of materials with general anisotropy, Phys. Rev. B 69, 094301 (2004).

[42] D. Yudistira, Y. Pennec, B. Djafari Rouhani, S. Dupont, and V. Laude. Non-radiative complete surface acoustic wave bandgap for finite-depth holey phononic crystal in lithium niobate, Appl. Phys. Lett. 100, 061912 (2012)

[43] A. Khelif, Y. Achaoui, S. Benchabane, V. Laude, and B. Aoubiza, Locally resonant surface acoustic wave band gaps in a two-dimensional phononic crystal of pillars on a surface. Phys. Rev. B 81, 214303 (2010).

[44] J. F. Robillard, A. Devos, and I. Roch-Jeune, Time-resolved vibrations of two-dimensional hypersonic phononic crystals, Phys. Rev. B 76, 092301 (2007)

[45] Y. Achaoui, A. Khelif, S. Benchabane, L. Robert, and V. Laude, Experimental observation of locally-resonant and Bragg band gaps for surface guided waves in a phononic crystal of pillars, Phys. Rev. B 83, 104201 (2011)

[46] D. Yudistira, A. Boes, B. Graczykowski, F. Alzina, L. Y. Yeo, C. M. Sotomayor Torres, and A. Mitchell, Nanoscale pillar hypersonic surface phononic crystals, Phys. Rev. B 94, 094304 (2016)

[47] C. Giannetti, B. Revaz, F. Banfi, M. Montagnese, G. Ferrini, F. Cilento, S. Maccalli, P. Vavassori, G. Oliviero, E. Bontempi, L. E. Depero, V. Metlushko, and F. Parmigiani, Thermomechanical behavior of 
surface acoustic waves in ordered arrays of nanodisks studied by near-infrared pump-probe diffraction experiments, Phys. Rev. B 76, 125413 (2007)

[48] B. J. Ash, S. R. Worsfold, P. Vukusic, and G. R. Nash, A highly attenuating and frequency tailorable annular hole phononic crystal for surface acoustic waves, Nature Communications 8, 174 (2017)

[49] S. Brûlé, E. H. Javelaud, S. Enoch, and S. Guenneau, Experiments on Seismic Metamaterials: Molding Surface Waves, Phys. Rev. Lett. 112, 133901 (2014)

[50] A. Colombi, P. Roux, S. Guenneau, P. Gueguen, and R. V. Craster, Forests as a natural seismic metamaterial: Rayleigh wave bandgaps induced by local resonances, Scientific Reports 6, 19238 (2016)

[51] M. Al Lethawe, M. Addouche, S. Benchabane, Vincent Laude, and Abdelkrim Khelif, Guidance of surface elastic waves along a linear chain of pillars, AIP Advances 6, 121708 (2016)

[52] S. Benchabane, R. Salut, O. Gaiffe, V. Soumann, M. Addouche, V. Laude, and A. Khelif, SurfaceWave Coupling to Single Phononic Subwavelength Resonators, Phys. Rev. Applied 8, 034016 (2017)

[53] U. Fano, Effects of configuration interaction on intensities and phase shifts, Phys. Rev. 124, 1866 (1961)

[54] D. Nardi, M. Travagliati, M. E. Siemens, Q. Li, M. M. Murnane, H. C. Kapteyn, G. Ferrini, F. Parmigiani, and F. Banfi, Probing Thermomechanics at the Nanoscale: Impulsively Excited Pseudosurface Acoustic Waves in Hypersonic Phononic Crystals, Nano Lett. 11, 4126 (2011),

[55] D. Nardi, M. Travagliati, M. M. Murnane, H. C. Kapteyn, G. Ferrini, C. Giannetti, and Francesco Banfi, Impulsively excited surface phononic crystals: a route toward novel sensing schemes, Sensors Journal IEEE 15, 5142 (2015).

[56] K.-J. Boller, A. Imamoglu, and S. E. Harris, Observation of electromagnetically induced transparency, Phys. Rev. Lett. 66, 2593 (1991)

[57] M. Fleischhauer, A. Imamoglu, and J. P. Marangos, Electromagnetically induced transparency: Optics in coherent media. Rev. Mod. Phys. 77, 633 (2005)

[58] G. C. Dyer, G. R. Aizin, S. J. Allen, A. D. Grine, D. Bethke, J. L. Reno, and E. A. Shaner, Induced transparency by coupling of Tamm and defect states in tunable terahertz plasmonic crystals, Nature Photonics 7, 925 (2013)

[59] T. F. Ciszek, T. H. Wang, X. Wu, R. W. Burrows, J. Alleman, C. R. Schwerdtfeger, T. Bekkedahl, Si thin layer growth from metal solutions on single-crystal and cast metallurgical-grade multicrystalline $\mathrm{Si}$ substrates, Photovoltaic Specialists Conference, Conference Record of the Twenty Third IEEE. (IEEE, New York, 1993), No. 93CH3283-9.

[60] K. Ohtsuka, A. Murai, T. Oizumi, T. Yoshida, T. Kurabayashi, K. Suto, and J. Nishizawa, Lowtemperature deposition of Si thin layer by gas-source molecular beam epitaxy, Journal of Vacuum Science \& Technology A: Vacuum, Surfaces, and Films 18, 48 (2000) 
[61] S. Anguiano, A. E. Bruchhausen, B. Jusserand, I. Favero, F. R. Lamberti, L. Lanco, I. Sagnes, A. Lemaître, N. D. Lanzillotti-Kimura, P. Senellart, and A. Fainstein, Micropillar Resonators for Optomechanics in the Extremely High 19-95 GHz Frequency Range, Phys. Rev. Lett. 118, 263901 (2017) [62] F. R. Lamberti, Q. Yao, L. Lanco, D. T. Nguyen, M. Esmann, A. Fainstein, P. Sesin, S. Anguiano, V. Villafañe, A. Bruchhausen, P. Senellart, I. Favero, and N. D. Lanzillotti-Kimura, Optomechanical properties of GaAs/AlAs micropillar resonators operating in the $18 \mathrm{GHz}$ range, Optics Express 25, 24437 (2017)

[63] M. Oudich, B. Djafari-Rouhani, B. Bonello, Y. Pennec and F. Sarry, Phononic crystal made of multilayered ridges on a substrate for Rayleigh waves manipulation, Crystals 7, 372 (2017) 